\title{
Urine exosome gene expression of cancer-testis antigens for prediction of bladder carcinoma
}

This article was published in the following Dove Press journal:

Cancer Management and Research

\section{Fatemeh Yazarlou' \\ Seyed Javad Mowla ${ }^{2}$ \\ Vahid Kholghi Oskooei ${ }^{3}$ \\ Elahe Motevaseli ${ }^{4}$ \\ Leila Farhady Tooli ${ }^{5}$ \\ Mandana Afsharpad ${ }^{6}$ \\ Leila Nekoohesh ${ }^{7}$ \\ Nafiseh Sadat Sanikhani ${ }^{4}$ \\ Soudeh Ghafouri-Fard ${ }^{3}$ \\ Mohammad Hossein \\ Modarressi'}

'Department of Medical Genetics, School of Medicine, Tehran University of Medical

Sciences, Tehran, Iran; ${ }^{2}$ Faculty of Biological

Sciences, Department of Genetics,

Tarbiat Modares University, Tehran, Iran;

${ }^{3}$ Department of Medical Genetics, Shahid

Beheshti University of Medical Sciences,

Tehran, Iran; ${ }^{4}$ Department of Molecular

Medicine, School of Advanced Technologies

in Medicine, Tehran University of Medical

Sciences, Tehran, Iran; ${ }^{5}$ Department of

Microbiology, School of Biology, College

of Science, Tehran University, Tehran, Iran;

${ }^{6}$ Cancer Control Research Center, Cancer

Control Foundation, Iran University of

Medical Sciences, Tehran, Iran; ${ }^{7}$ Department

of Medical Biotechnology, School of

Advanced Technologies in Medicine, Tehran

University of Medical Sciences, Tehran, Iran

Correspondence: Soudeh Ghafouri-Fard Department of Medical Genetics, Shahid Beheshti University of Medical Sciences, Daneshjoo Boulevard, Velenjak, Tehran 19857-I7443, Iran

Email s.ghafourifard@sbmu.ac.ir

Mohammad Hossein Modarressi

Department of Medical Genetics, School of Medicine, Tehran University of Medical Sciences, Keshavarz Boulevard, Tehran |416753955, Iran

Email modaresi@tums.ac.ir
Background: Exosomes have been regarded as emerging tools for cancer diagnosis. Tumorderived exosomes contain molecules that enhance cancer progression and affect immune responses.

Material and methods: In the present study, we evaluated expression of seven cancer-testis antigens (CTAs) that are regarded as putative biomarkers and immunotherapeutic targets along with NMP22 in urinary exosomes of bladder cancer patients, healthy subjects and patients affected with nonmalignant urinary disorders.

Results: Exosomal expression of $M A G E-B 4$ was significantly higher in bladder cancer patients compared with normal samples (expression ratio $=2.68, P=0.01$ ). However, its expression was lower in bladder cancer patients compared with benign prostate hyperplasia (BPH) patients (expression ratio $=0.17, P=0.01$ ). Exosomal expression of $N M P 22$ was significantly higher in bladder cancer patients compared with BPH patients (expression ratio=9.22, $P=0.02$ ). Expressions of other genes were not significantly different between bladder cancer patients and normal/ nonmalignant samples. We found significant correlation between $M A G E-A 3$ and $M A G E-B 4$ expressions in exosomes obtained from controls. In addition, TSGA10 expression was correlated with expression of NMP22 in both cancer patients and controls.

Conclusion: The present study provides evidences for differential expression of CTAs in urinary exosomes of bladder cancer patients and urogenital disorders and warrants further studies for assessment of their significance in cancer diagnosis and immunotherapeutic approaches.

Keywords: cancer-testis antigen, bladder cancer, exosome

\section{Introduction}

Exosomes as natural extracellular nanoparticles have been detected in nearly all biofluids including urine. Assessment of exosome contents has led to identification of pathophysiological features of the corresponding tissues. Consequently, extensive efforts have been made to apply urinary exosome content as biomarkers with the hope to replace the invasive tissue biopsy. ${ }^{1}$ The lipids, mRNA, and microRNA content of exosomes might participate in the pathogenesis of human disorders through altering intercellular communication. ${ }^{2}$ In addition, cancer cell-derived exosomes can change tumor microenvironment in favor of cancer cell growth and arrange subsequent steps in the promotion of metastasis and chemoresistance. ${ }^{3}$ Bladder cancer cell-originated exosomes have been shown to suppress tumor cell apoptosis via induction of Akt and ERK pathway genes. ${ }^{4}$ Moreover, expression of an extracellular matrix-associated glycoprotein in urinary exosomes of bladder cancer patients has been demonstrated to enhance invasive properties of cancer cells and endothelial cells. ${ }^{5}$ Cancer-testis 
antigens (CTAs) are among putative biomarkers in bladder cancer patients whose expressions are associated with clinical features of the corresponding tumors. ${ }^{6}$ They are also involved in the critical aspects of tumor proliferation, migration, and invasion. ${ }^{7}$ These properties along with their selective expression in tumoral tissues despite their absence in normal tissues make them appropriate targets in cancer immunotherapy. ${ }^{8}$ Consequently, their expression in cancer-derived exosomes might be involved in the pathogenesis of cancer on one hand and might be applied as therapeutic target on the other hand. The latter is enforced by the growing application of exosomes in specific delivery of drugs and other therapeutic agents to cancer cells. ${ }^{3}$ Based on our previous detection of CTA expression in urinary exfoliated cells (UEC) of patients with transitional cell carcinoma (TCC), ${ }^{6}$ we selected seven CTAs to assess their expression in urinary exosomes isolated from TCC patients and controls. As NMP22 is regarded as a marker of urothelial cell death whose expression is increased in the urine of TCC patients, ${ }^{9}$ we also assessed expression of NMP22 in urinary exosomes of all study participants.

\section{Materials and methods}

\section{Sample collection}

The Ethical Committee of Tehran University of Medical Sciences approved this study (ethical code: 23968-51-03-93). The study participants provided written informed consent, which was conducted in accordance with the Declaration of Helsinki. Random urine samples were collected from 59 patients with TCC, 24 healthy volunteers, 11 patients with bladder stone, six patients with obstructive uropathy, and eight patients with benign prostate hyperplasia (BPH). Urine samples were stored at $4^{\circ} \mathrm{C}$ until further assessments.

\section{Urine exosome isolation}

Urine exosomes were isolated by spin column protocol as provided in Norgen's Urine Exosome RNA Isolation Kit (BIOTEK Corporation, Thorold, ON, Canada). This kit establishes an all-in-one system for the exosome isolation and isolation of exosomal RNA from urine.

\section{Urine exosome confirmation}

The size and shape of exosomes were confirmed by western blotting, dynamic light scattering (DLS) assessments, and electron microscopy.

\section{Western blotting}

Urinary exosome were subjected to western blot analysis with antibodies against exosomal marker protein CD63. In brief, the protein concentration of the preparations was evaluated using Bradford protein assay. BSA was applied as the standard sample. Samples were incubated for 5 minutes at $37^{\circ} \mathrm{C}$ and separated on $10 \%$ precasted gel. Subsequently, they were transferred to nitrocellulose membranes and blocked overnight ( $5 \%$ milk and $0.05 \%$ Tween-20 in PBS). Then they were incubated with primary antibody (Santa Cruz Biotechnology, Dallas, TX, USA.) for 1 hour, washed by PBS, and finally incubated with secondary HRP-conjugated antibody (SinaClon, Tehran, Iran). The corresponding immunoreactive bands were visualized using chemiluminescent detection system. The molecular weights of proteins were assessed using the prestained protein ladder (SinaClon).

\section{DLS assessments}

The exosomes were sized by DLS using a Zetasizer Nano ZS (Malvern Instruments, Malvern, UK) according to the company guidelines.

\section{Electron microscopy}

A portion of the purified exosomes was fixed in $2.5 \%$ glutaraldehyde, dehydrated with mounting grades of ethanol, vacuum dried on a glass surface, and sputter coated with gold. The size and shape of the exosomes were evaluated using scanning electron microscopy (SEM) (The QUANTA SEM system, FEI Company, Hillsboro, OR, USA).

\section{Exosomal RNA isolation}

RNA was isolated from urine exosomes using Urine Exosome RNA Isolation Kit (Norgen, BIOTEK Corporation) according to manufacturer's instructions. This kit uses an all-in-one system for the concentration and isolation of exosomal RNA from biological samples. After binding of the urinary exosomes to a patented resin, RNA is refined from the exosome by means of a column-based method.

\section{Quantitative real-time PCR analysis}

The first strand cDNA was synthesized from RNA samples using PrimeScript ${ }^{\mathrm{TM}}$ RT reagent Kit (Takara, Tokyo, Japan). The relative transcript levels of CTA genes in urine exosomes were quantified in the rotor gene 6000 Corbett RealTime PCR System using RealQ Plus 2x Master Mix Green (Ampliqon, Odense, Denmark). Gene expression analyses were performed in a total volume of $30 \mu \mathrm{L}$. $5 \mathrm{~S}$ rRNA was used to normalize expression levels. All experiments were performed in duplicates. A cDNA pool was prepared for primary assessment of CTA gene expressions. CTA genes with no expression in this sample were excluded from further 
studies. The nucleotide sequence of primers used in expression analyses are shown in Table 1.

\section{Statistical methods}

SPSS v.18.0 (IBM Corp., Armonk, NY, USA) was used for statistical analyses. The relative expression of each gene was quantified using Efficiency ${ }^{\wedge} \mathrm{CT}_{\text {normalizer gene }} /$ Efficiency $^{\wedge} \mathrm{CT}$ target gene equation. The magnitude of expression of each gene between cancerous and control samples was described as fold change value and was calculated by dividing the obtained values in cancerous sample to the corresponding value of control sample. The association between clinicopathological data and relative expression of genes in TCC patients was calculated using independent $t$-test. Correlation between expression levels of genes in urine samples was assessed using Pearson correlation coefficient. The level of significance was set at $P<0.05$ in all analyses. The receiver operating characteristic (ROC) curve was designed to appraise the suitability of gene expression levels for differentiation of TCC status from non- malignant conditions. The Youden index (j) was applied to obtain the most difference between sensitivity (true-positive rate) and 1 - specificity (false-positive rate). The validity of transcript level of each gene for diagnosis of bladder cancer was described through calculation of the area under curve (AUC) values. AUC values were judged using the following method: $0.90-1=\operatorname{excellent}(\mathrm{A}), 0.80-0.90=\operatorname{good}(\mathrm{B}), 0.70-$ $0.80=$ fair $(C), 0.60-0.70=$ poor $(D)$, and $0.50-0.60=$ fail $(F)$.

\section{Results \\ General data of patients}

The general characteristics of study participants are summarized in Table 2 .

\section{Confirmation of exosome size and morphology}

Assessment of isolated particles with SEM confirmed that we could effectively isolate exosomes with acceptable quality regarding their size range and morphology (Figure 1).

Table I The nucleotide sequence of primers used in expression analyses

\begin{tabular}{|l|l|l|}
\hline Gene name & Forward primer & Reverse primer \\
\hline NMP22 & AGATGACACTCCACGCCACC & TGCTGTCCCTCTTCAGTGCC \\
\hline MAGE-A3 & GTCGTCGGAAATTGGCAGTAT & TGGGGTCCACTTCCATCA \\
\hline MAGE-B4 & ACGAAGATGTTAGTGCAGTTCC & GTGCGCTGAGAGACTTTCC \\
\hline TSGA-I0 & ATGAGCGCCATTTGGCAGAA & GGGTAATTTCTTCCTGTGCCTG \\
\hline AKAP4 & TAACGTGCCCATGCTCTACT & ACCTCCTACACTGTACCCCT \\
\hline SYCPI & ACGGGAAGAAACCAGGCAAG & GGAATTCTCAGCTTGCACACG \\
\hline OY-TES-I & TGCTCCAACCTCCCTTATGC & CGTGGTGGGTGAGACTTCAG \\
\hline NY-ESO-I & TATGTTGCCGGACACAGTGA \\
\hline $5 S$ rRNA & TCAGGCTGAATGGATGCTG & AGCCTACAGCACCCGGTATT \\
\hline
\end{tabular}

Table 2 The general characteristics of study participants

\begin{tabular}{|c|c|c|c|c|c|}
\hline Study groups & \multicolumn{2}{|l|}{ Number of samples } & \multirow{2}{*}{\multicolumn{2}{|c|}{$\begin{array}{l}\text { Variables } \\
\text { Age (mean } \pm S D \text { [range]) }\end{array}$}} & Values \\
\hline \multirow[t]{7}{*}{ TCC patients } & \multirow[t]{7}{*}{59} & & & & $61.28 \pm 13.01(33-84)$ \\
\hline & & \multirow[t]{2}{*}{ Smoking } & Negative & $16(27.1 \%)$ & \\
\hline & & & Positive & 43 (72.8\%) & \\
\hline & & \multirow{2}{*}{$\begin{array}{l}\text { Opium } \\
\text { addiction }\end{array}$} & Negative & $30(50.8 \%)$ & \\
\hline & & & Positive & $29(49.1 \%)$ & \\
\hline & & \multirow[t]{2}{*}{ Grade } & High grade & $34(57.6 \%)$ & \\
\hline & & & Low grade & $25(42.3 \%)$ & \\
\hline \multirow[t]{7}{*}{ Controls } & \multirow[t]{3}{*}{ Total } & \multirow[t]{3}{*}{49} & \multicolumn{2}{|c|}{ Age (mean \pm SD [range]) } & $64.42 \pm 15.53(31-88)$ \\
\hline & & & \multirow[t]{2}{*}{ Smoking } & Negative & $30(61.2 \%)$ \\
\hline & & & & Positive & $19(38.7 \%)$ \\
\hline & Normal & 24 & \multicolumn{2}{|c|}{ Age (mean \pm SD [range]) } & $68 \pm 13.56(39-88)$ \\
\hline & Obstructive uropathy & 6 & \multicolumn{2}{|c|}{ Age (mean \pm SD [range]) } & $42.5 \pm 8.26(33-52)$ \\
\hline & Bladder stone & 11 & \multicolumn{2}{|c|}{ Age (mean \pm SD [range]) } & $55.42 \pm 155.55(3 I-84)$ \\
\hline & $\mathrm{BPH}$ & 8 & \multicolumn{2}{|c|}{ Age (mean $\pm S D$ [Range]) } & $73 \pm 7.48(54-8 I)$ \\
\hline
\end{tabular}

Abbreviations: $\mathrm{BPH}$, benign prostate hyperplasia; TCC, transitional cell carcinoma. 


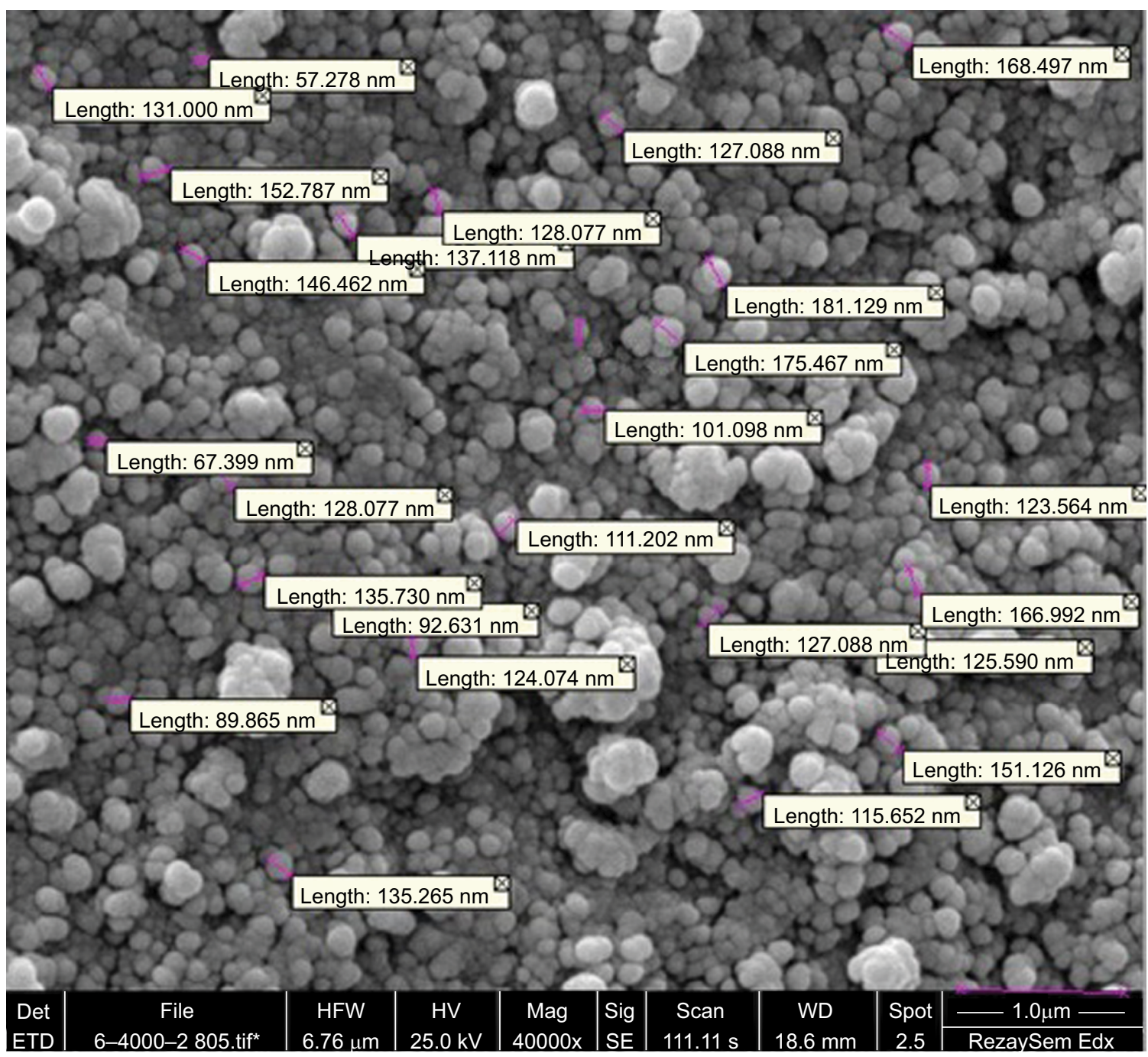

Figure I Scanning electron micrographs of fixed and dehydrated exosomes isolated from urine samples.

The results were also confirmed by DLS assessment (Figure 2). In addition, western blot analysis of samples showed the expression of CD63 as a common marker of exosomes.

\section{Expression of CTA genes in urinary exosomes}

After confirmation of exosome isolation through SEM and western blot analyses, we assessed the expression of seven CTAs along with $N M P 22$ in all samples. SYCP1, OY-TES, NY-ESO-1, and AKAP4 expressions were not detected in the cDNA pool prepared from all exosomal samples, and so these genes were excluded from further steps. Other genes were detected in exosomes of both TCC patients and nonmalignant conditions. Exosomal expression of MAGE-B4 was significantly higher in TCC patients compared with normal samples (expression ratio $=2.68, P=0.01$ ). However, its expression was lower in TCC patients compared with BPH patients (expression ratio $=0.17, P=0.01$ ). Exosomal expression of $N M P 22$ was significantly higher in TCC patients compared with $\mathrm{BPH}$ patients (expression ratio $=9.22, P=0.02$ ). Expressions of other genes were not significantly different between TCC patients and normal/nonmalignant samples. Table 3 shows relative expression of genes in urinary exosomes of TCC patients compared with nonmalignant conditions.

\section{Associations between exosomal expression of CTA genes and patients' clinicopathological features}

No significant association was found between relative expression of genes in urinary exosomes of TCC patients and clinicopathological data (Table 4). 


$\begin{array}{rlllll} & & & \text { Diam. }(\mathrm{nm}) & \text { \% number } & \text { Width }(\mathrm{nm}) \\ \text { Z-Average (d.nm): } & 240 & \text { Peak 1: } & 57.8 & 100.0 & 19.3 \\ \text { Pdl } & 0.336 & \text { Peak 2: } & 0.00 & 0.0 & 0.00 \\ \text { Intercept } & 0.840 & \text { Peak 3: } & 0.00 & 0.0 & 0.00\end{array}$

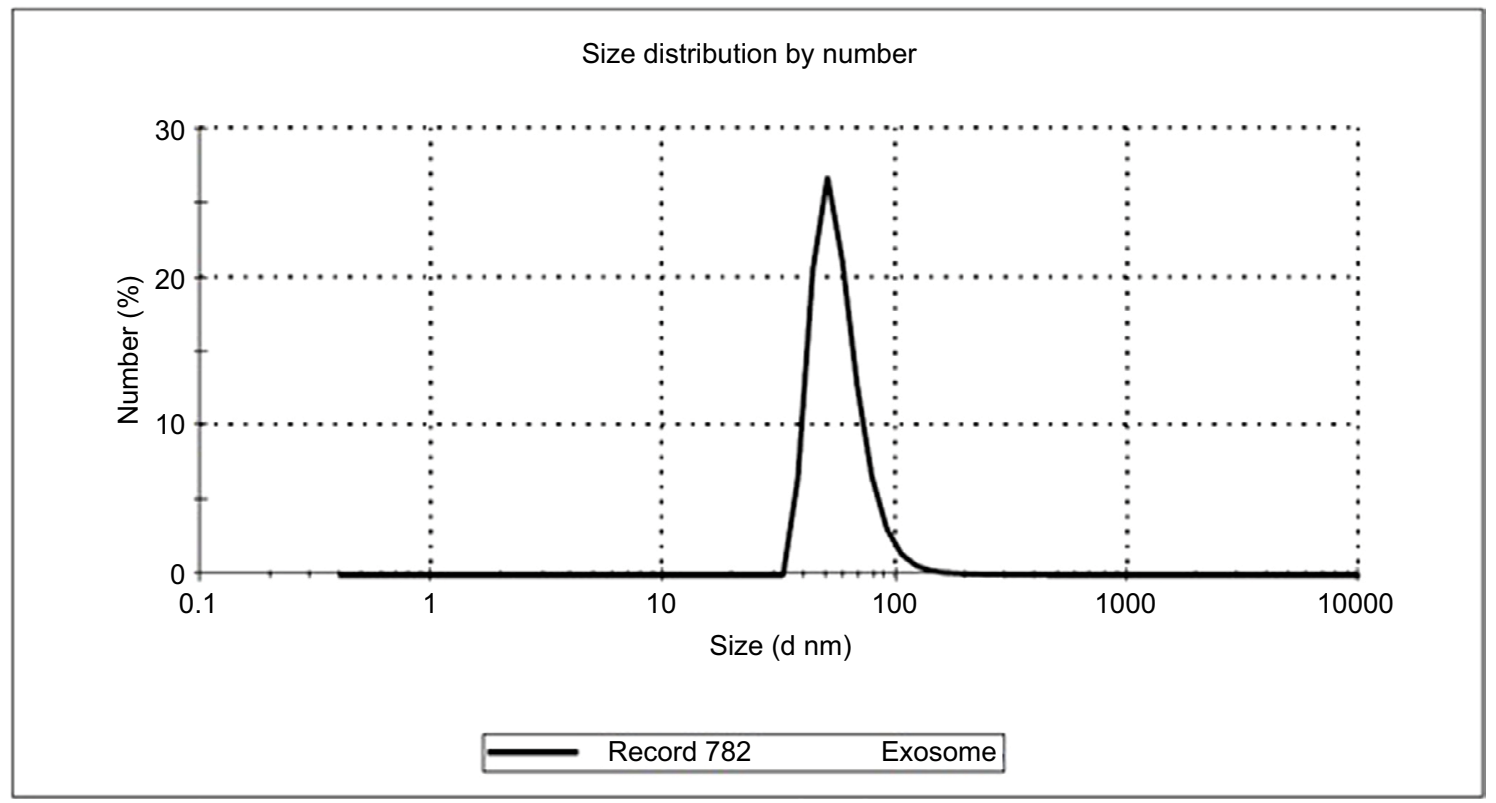

Figure 2 Size distribution of exosomes by volume as demonstrated by Zetasizer Nano ZS (Malvern Instruments).

Table 3 Relative expression of genes in urinary exosomes of TCC patients compared with nonmalignant conditions

\begin{tabular}{|c|c|c|c|c|c|}
\hline Gene names & $\begin{array}{l}\operatorname{TCC}(n=59) \text { vs } \\
\text { nonmalignant } \\
\text { conditions }(n=49)\end{array}$ & $\begin{array}{l}\operatorname{TCC}(n=59) \\
\text { vs normal } \\
\text { samples }(n=24)\end{array}$ & $\begin{array}{l}\operatorname{TCC}(n=59) \\
\text { vs obstructive } \\
\text { uropathy }(n=6)\end{array}$ & $\begin{array}{l}\text { TCC }(n=59) \\
\text { vs bladder } \\
\text { stone }(n=I I)\end{array}$ & $\begin{array}{l}\operatorname{TCC}(n=59) \\
\text { vs BPH }(n=8)\end{array}$ \\
\hline \multicolumn{6}{|l|}{ MAGE-A3 } \\
\hline Expression ratio & 1.26 & 1.39 & 1.1 & 1.17 & 1.11 \\
\hline$P$-value & 0.32 & 0.25 & 0.87 & 0.64 & 0.82 \\
\hline \multicolumn{6}{|l|}{ MAGE-B4 } \\
\hline Expression ratio & 1.3 & 2.68 & 0.71 & 1.09 & 0.17 \\
\hline$P$-value & 0.43 & 0.01 & 0.6 & 0.87 & 0.01 \\
\hline \multicolumn{6}{|l|}{ TSGAIO } \\
\hline Expression ratio & 1.32 & 2.18 & 0.04 & 2.86 & 1.1 \\
\hline$P$-value & 0.37 & 0.11 & 0.25 & 0.35 & 0.7 \\
\hline \multicolumn{6}{|l|}{ NMP22 } \\
\hline Expression ratio & 0.9 & 0.47 & 0.37 & 1.09 & 9.22 \\
\hline$P$-value & 0.78 & 0.15 & 0.28 & 0.92 & 0.02 \\
\hline
\end{tabular}

Abbreviations: $\mathrm{BPH}$, benign prostate hyperplasia; TCC, transitional cell carcinoma.

\section{Correlations between exosomal expressions of CTA genes}

We assessed correlations between expression levels of mentioned genes in urinary exosomes of both TCC patients and nonmalignant conditions. We found significant correlation between $M A G E-A 3$ and $M A G E-B 4$ expressions in exosomes obtained from controls. In addition, TSGA10 expression was correlated with expression of NMP22 in both TCC patients and controls. Table 5 shows the results of correlation analysis between relative expressions of genes in urinary exosomes.

\section{ROC curve analysis}

As we demonstrated significant overexpression of $M A G E-B 4$ in urinary exosomes of TCC patients compared with normal 
Table 4 Associations between relative expression of genes in urinary exosomes of TCC patients and clinicopathological data

\begin{tabular}{|c|c|c|c|c|c|c|c|c|}
\hline $\begin{array}{l}\text { Demographic } \\
\text { data }\end{array}$ & MAGE-A3 & $P$-value & MAGE-B4 & $P$-value & TSGAIO & $P$-value & NMP22 & $P$-value \\
\hline \multicolumn{2}{|l|}{ Age } & 0.62 & & 0.14 & & 0.23 & & 0.22 \\
\hline$<60$ years & $0.02 \pm 0.03$ & & $0.01 \pm 0.02$ & & $107.34 \pm 442.58$ & & $0.03 \pm 0.12$ & \\
\hline$\geq 60$ years & $0.03 \pm 0.14$ & & $0.005 \pm 0.009$ & & $0.02 \pm 0.11$ & & $0.0007 \pm 0.001$ & \\
\hline \multicolumn{2}{|l|}{ Cigarette smoking } & 0.47 & & 0.91 & & 0.58 & & 0.07 \\
\hline Yes & $0.03 \pm 0.12$ & & $0.008 \pm 0.0 \mathrm{I}$ & & $57.02 \pm 322.58$ & & $0.0006 \pm 0.00 \mathrm{I}$ & \\
\hline No & $0.01 \pm 0.01$ & & $0.007 \pm 0.01$ & & $0.06 \pm 0.18$ & & $0.04 \pm 0.04$ & \\
\hline \multicolumn{2}{|l|}{ Opium addiction } & 0.32 & & 0.95 & & 0.37 & & 0.29 \\
\hline Yes & $0.05 \pm 0.17$ & & $0.009 \pm 0.02$ & & $101.38 \pm 530.11$ & & $0.0006 \pm 0.001$ & \\
\hline No & $0.01 \pm 0.02$ & & $0.009 \pm 0.01$ & & $0.003 \pm 0.0 \mathrm{I}$ & & $0.03 \pm 0.12$ & \\
\hline \multicolumn{2}{|l|}{ Grade } & 0.31 & & 0.27 & & 0.44 & & 0.2 \\
\hline High grade & $0.04 \pm 0.14$ & & $0.006 \pm 0.009$ & & $72.99 \pm 364.96$ & & $0.00 \mathrm{I} \pm 0.002$ & \\
\hline Low grade & $0.0 \mathrm{I} \pm 0.0 \mathrm{I}$ & & $0.01 \pm 0.02$ & & $0.003 \pm 0.01$ & & $0.03 \pm 0.13$ & \\
\hline
\end{tabular}

Note: Relative expression levels were calculated using Efficiency^ $\mathrm{CT}_{\text {normalizer gene }} / \mathrm{Efficiency}^{\wedge} \mathrm{CT}_{\text {target gene }}$ formula and data are presented as mean $\pm \mathrm{SD}$.

Abbreviation: TCC, transitional cell carcinoma.

Table 5 Pearson correlation coefficient values between relative expression of genes in urinary exosomes of TCC patients and controls

\begin{tabular}{|l|l|l|l|l|}
\hline \multicolumn{2}{|l|}{ Demographic data } & NMP22 & TSGA I0 & MAGE-B4 \\
\hline MAGE-A3 & $\begin{array}{l}\text { TCC } \\
\text { samples }\end{array}$ & 0.02 & 0.03 & 0.04 \\
\hline \multirow{3}{*}{ MAGE-B4 } & Controls & 0.007 & 0.01 & $0.23^{* *}$ \\
\hline & $\begin{array}{l}\text { TCC } \\
\text { samples }\end{array}$ & -0.02 & -0.02 & \\
\cline { 2 - 5 } & Controls & 0.05 & -0.02 & \\
\hline TSGA10 & $\begin{array}{l}\text { TCC } \\
\text { samples }\end{array}$ & $0.12^{*}$ & & \\
\cline { 2 - 5 } & Controls & $0.12^{*}$ & \multicolumn{2}{|l|}{} \\
\hline
\end{tabular}

Note: Relative expression levels were calculated using Efficiency^ $\mathrm{CT}_{\text {normalizer gene }}{ }^{\prime}$ Efficiency^ $\mathrm{CT}_{\text {targe gene }}$ formula. $* P<0.05, * * P<0.01$.

Abbreviation: TCC, transitional cell carcinoma.

males, we assessed the performance of transcript levels of this gene in diagnosis of cancer status using ROC curve analysis (Figure 3). Transcript levels of this gene could be used as a diagnostic tool for bladder cancer with sensitivity and specificity of $71.7 \%$ and $66.7 \%$, respectively (Table 6).

\section{Discussion}

In the present study, we isolated and purified urinary exosomes from samples obtained from TCC patients as well as other urogenital disorders. Size, morphology, and expression of CD63 as a protein hallmark in these exosomes were similar to those of exosomes obtained from other biofluids. ${ }^{4,10} \mathrm{We}$ further assessed expression a mini-set of CTAs in the isolated exosomes and demonstrated expression of three CTAs namely $M A G E-A 3, M A G E-B 4$, and TSGA10 in urinary exosomes of TCC patients and nonmalignant conditions. The unique expression pattern of CTAs in immune-privileged sites and their re-expression in a wide range of cancers have potentiated them as a cancer vaccine candidate. ${ }^{11}$ Moreover, most of CTAs are considered as intrinsically disordered proteins (IDPs). Contrary to typical proteins that have unique threedimensional folding, IDPs could adopt different conformations to expand their interacting partners. This flexibility let them to be key players of various cellular processes acting as a hub in signaling pathways and cell cycle progression or act as a platform to assemble other protein compartments. ${ }^{12}$

$M A G E-A 3, M A G E-B 4$, and TSGA10 were among the most specifically expressed CTAs in TCC samples as revealed in our previous study. ${ }^{6}$ Moreover, MAGE-A3 expression and spontaneous immune responses against the encoded protein have been detected in several human malignancies. Such observations have led to establishment of several clinical trials of MAGE-A3 vaccines in cancer patients with preliminary promising results. ${ }^{8}$

On the other hand, we could not detect SYCP1, OY-TES, NY-ESO-1, and AKAP4 expressions in urinary exosomes isolated from TCC patients or nonmalignant conditions. $N Y$ ESO-1 has been among the most frequently expressed CTAs in bladder cancer tissues. ${ }^{13}$ The existence of spontaneous humoral and cellular immune responses in cancer patients has placed this CTA in the top of the list of CTAs being applied in immunotherapeutic clinical trials. ${ }^{7}$ We could not previously detect expression of AKAP4 in either UECs or tumoral tissues of TCC patients by conventional RT-PCR. ${ }^{6}$ However, to the best of our knowledge expressions of SYCP1, $O Y-T E S$, and NY-ESO-1 have not been assessed in UECs of TCC patients yet. Considering the importance of urinary exosomes and UECs as two separate tools for noninvasive 


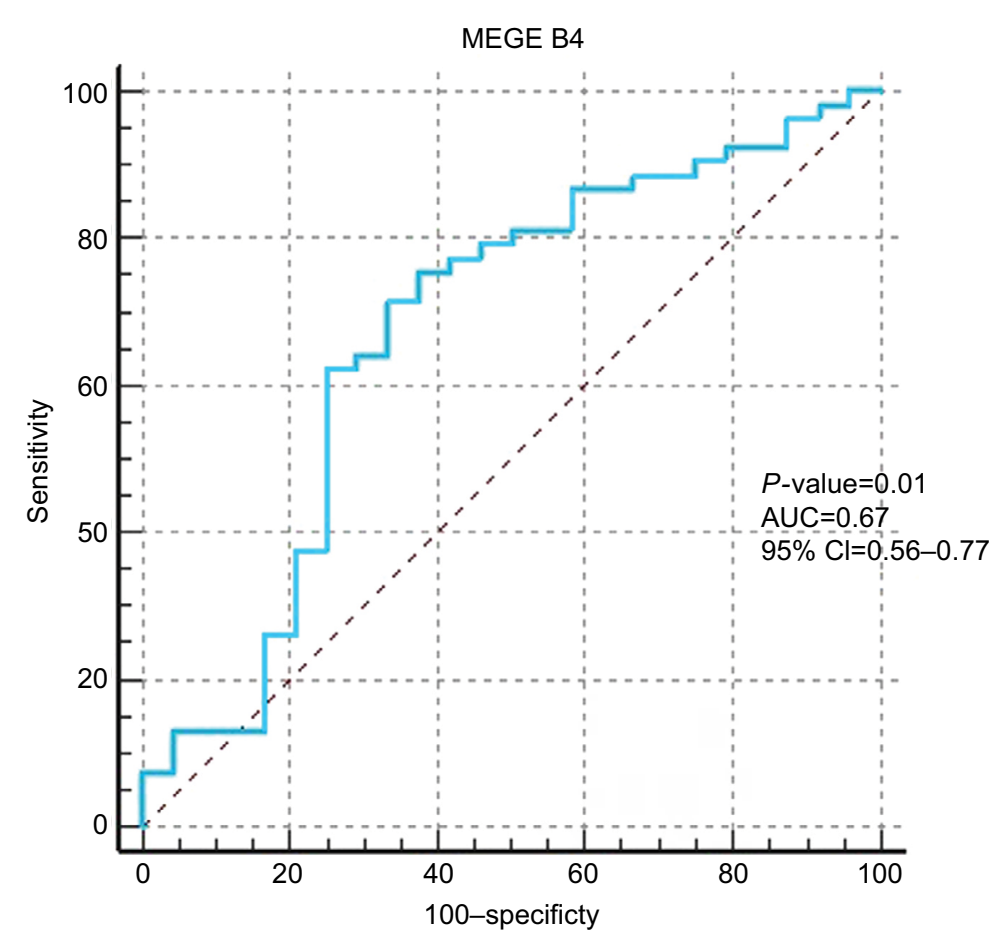

Figure 3 The results of ROC curve analysis of performance of MAGE-B4 transcript levels in urinary exosomes for diagnosis of bladder cancer. Abbreviations: AUC, area under the curve; ROC, receiver operating characteristic.

Table 6 The results of ROC curve analysis

\begin{tabular}{|l|l|l|l|l|l|l|l|}
\hline Gene name & Estimate criterion & AUC & $\mathbf{J}^{\mathrm{a}}$ & Sensitivity & Specificity & $\mathbf{9 5 \%} \mathbf{C l}$ & $\boldsymbol{P}_{\text {-value }} \mathbf{b}^{\mathrm{b}}$ \\
\hline MAGE-B4 & $\leq \mathrm{II} .02$ & 0.67 & 0.38 & 71.7 & 66.7 & $0.56-0.77$ & 0.01 \\
\hline
\end{tabular}

Note: a Youden index, ${ }^{b}$ significance level of $P$ (area=0.5), estimate criterion: optimal cutoff point for gene expression.

Abbreviations: AUC, area under curve; ROC, receiver operating characteristic.

detection of bladder cancer, parallel assessment of these two sources might facilitate design of novel diagnostic panels.

We demonstrated higher expression of $M A G E-B 4$ in urinary exosomes of TCC patients compared with normal samples, which is in line with the proposed role for MAGE proteins in inhibition of apoptosis and enhancement of tumor cell survival. ${ }^{14}$ However, $M A G E-B 4$ expression was lower in TCC patients compared with BPH patients. We previously demonstrated specific expression of $M A G E-B 4$ in TCC tissues and UEC samples of patients compared with noncancerous tissues and control UEC samples. ${ }^{6}$ Consequently, $M A G E-B 4$ seems to have a parallel expression in both UECs and exosomes, which facilitates its application as a diagnostic biomarker. In spite of frequent expression of $M A G E$ genes in tumoral tissues, the evaluation of function of $M A G E$ genes in normal development has been precluded by absence of domains with noticeable homology to a definite motif or domain with acknowledged function. ${ }^{15}$ Necdin, as one putative member of the MAGE family, is expressed in neurons where it interacts with viral transforming proteins and the transcription factor E2F1 leading to inhibition of E2F1-dependent transcription. ${ }^{16,17}$ The observed different pattern of $M A G E-B 4$ expression between TCC and BPH samples might be attributed to low expression of $\mathrm{E} 2 \mathrm{~F} 1$ in $\mathrm{BPH}^{18}$ in spite of its activation in bladder cancer cells. ${ }^{19}$ Few studies have reported a causal link between the presence of viral transforming proteins and development of both primary urothelial carcinoma and distant metastases. ${ }^{20}$ Future studies should focus on assessment of interactions between MAGE-B4 and viral transforming proteins and the significance of it in immunotherapy of TCC.

We also detected higher exosomal expression of NMP22 in TCC patients compared with BPH patients, which is in line with the frequently reported elevated levels of NMP22 protein in urine samples of TCC patients. ${ }^{21}$ 
In spite of the observed significant upregulation of $M A G E-B 4$ in TCC exosomes, transcript levels of this gene had the sensitivity and specificity values of $71.7 \%$ and $66.7 \%$, respectively. Future studies are necessary to assess its suitability to be included in a panel of urinary exosome transcripts for diagnosis of bladder cancer.

Finally, we found significant correlation between $M A G E$ $A 3$ and $M A G E-B 4$ expressions in exosomes obtained from controls, which is consistent with the presence of a common epigenetic mechanism for expression of $M A G E$ genes. ${ }^{14}$ In addition, TSGA10 expression correlated with expression of NMP22 in both TCC patients and controls. TSGA10 is a CTA with widespread expression in human malignancies. However, its forced expression in HeLa cells has disrupted HIF-1 $\alpha$ axis and suppressed tumor angiogenesis and metastasis. ${ }^{22} \mathrm{On}$ the other hand, NMP22 is a nuclear matrix protein, which is released from cells during apoptosis. ${ }^{23}$ The observed correlation between TSGA10 and NMP22 expressions in both cases and controls might reflect a functional link between these two transcripts, which should be evaluated in future studies.

In brief, in the present study we could demonstrate expression of three CTA transcripts in urinary exosomes and assess their potential in TCC diagnosis. Based on the differential expression of some CTAs in urological disorders, they might be applied as diagnostic markers in these kinds of disorders. However, the relative small sample size has been a limitation of our study. Future studies are needed to evaluate this hypothesis in larger sample sizes.

\section{Acknowledgments}

The current study was supported by a grant from Tehran University of Medical Sciences.

\section{Disclosure}

The authors report no conflicts of interest in this work.

\section{References}

1. Street JM, Koritzinsky EH, Glispie DM, Star RA, Yuen PS. Urine Exosomes: An Emerging Trove of Biomarkers. Adv Clin Chem. 2017;78:103-122.

2. Valadi H, Ekström K, Bossios A, Sjöstrand M, Lee JJ, Lötvall JO. Exosome-mediated transfer of mRNAs and microRNAs is a novel mechanism of genetic exchange between cells. Nat Cell Biol. 2007;9(6):654-659.
3. Sun W, Luo JD, Jiang H, Duan DD. Tumor exosomes: a double-edged sword in cancer therapy. Acta Pharmacol Sin. 2018;39(4):534-541.

4. Yang L, Wu XH, Wang D, Luo CL, Chen LX. Bladder cancer cellderived exosomes inhibit tumor cell apoptosis and induce cell proliferation in vitro. Mol Med Rep. 2013;8(4):1272-1278.

5. Beckham CJ, Olsen J, Yin PN, et al. Bladder cancer exosomes contain EDIL-3/Del1 and facilitate cancer progression. J Urol. 2014;192(2):583-592.

6. Afsharpad M, Nowroozi MR, Mobasheri MB. Cancer-testis antigens as new candidate diagnostic biomarkers for transitional cell carcinoma of bladder. Pathol Oncol Res. 2017.

7. Esfandiary A, Ghafouri-Fard S. New York esophageal squamous cell carcinoma-1 and cancer immunotherapy. Immunotherapy. 2015;7(4):411-439.

8. Esfandiary A, Ghafouri-Fard S. MAGE-A3: an immunogenic target used in clinical practice. Immunotherapy. 2015;7(6):683-704.

9. Leiblich A. Recent Developments in the Search for Urinary Biomarkers in Bladder Cancer. Curr Urol Rep. 2017;18(12):100.

10. Andre F, Schartz NE, Movassagh M, et al. Malignant effusions and immunogenic tumour-derived exosomes. Lancet. 2002;360(9329):295-305.

11. Ghafouri-Fard S, Ghafouri-Fard S. Immunotherapy in nonmelanoma skin cancer. Immunotherapy. 2012;4(5):499-510.

12. Rajagopalan K, Mooney SM, Parekh N, Getzenberg RH, Kulkarni P. A majority of the cancer/testis antigens are intrinsically disordered proteins. J Cell Biochem. 2011;112(11):3256-3267.

13. Fradet Y, Picard V, Bergeron A, Larue H. Cancer-testis antigen expression in bladder cancer. Prog Urol. 2005;15(6 Suppl 1):1303-1313.

14. Yang B, Wu J, Maddodi N, Ma Y, Setaluri V, Longley BJ. Epigenetic control of MAGE gene expression by the KIT tyrosine kinase. J Invest Dermatol. 2007;127(9):2123-2128.

15. Osterlund C, Töhönen V, Forslund KO, Nordqvist K. Mage-b4, a novel melanoma antigen (MAGE) gene specifically expressed during germ cell differentiation. Cancer Res. 2000;60(4):1054-1061.

16. Taniura H, Taniguchi N, Hara M, Yoshikawa K. Necdin, a postmitotic neuron-specific growth suppressor, interacts with viral transforming proteins and cellular transcription factor E2F1. J Biol Chem. 1998;273(2):720-728.

17. Kuwako K, Taniura H, Yoshikawa K. Necdin-related MAGE proteins differentially interact with the E2F1 transcription factor and the p75 neurotrophin receptor. J Biol Chem. 2004;279(3):1703-1712.

18. Davis JN, Wojno KJ, Daignault S, et al. Elevated E2F1 inhibits transcription of the androgen receptor in metastatic hormone-resistant prostate cancer. Cancer Res. 2006;66(24):11897-11906.

19. Lin ZR, Wang MY, He SY, Cai ZM, Huang WR. TACC3 transcriptionally upregulates E2F1 to promote cell growth and confer sensitivity to cisplatin in bladder cancer. Cell Death Dis. 2018;9(2):72.

20. van Aalderen MC, Yapici Ü, van der Pol JA, et al. Polyomavirus BK in the pathogenesis of bladder cancer. Neth J Med. 2013;71(1):26-28.

21. Sánchez-Carbayo M, Herrero E, Megías J, Mira A, Soria F. Evaluation of nuclear matrix protein 22 as a tumour marker in the detection of transitional cell carcinoma of the bladder. BJU Int. 1999;84(6):706-713.

22. Mansouri K, Mostafie A, Rezazadeh D, Shahlaei M, Modarressi $\mathrm{MH}$. New function of TSGA10 gene in angiogenesis and tumor metastasis: a response to a challengeable paradox. Hum Mol Genet. 2016;25(2):233-244.

23. Szymańska B, Sawicka E, Guzik A, Zdrojowy R, Długosz A. The Diagnostic Value of Nuclear Matrix Proteins in Bladder Cancer in the Aspect of Environmental Risk from Carcinogens. Biomed Res Int. 2017;2017:9643139-11. 
Cancer Management and Research

\section{Publish your work in this journal}

Cancer Management and Research is an international, peer-reviewed open access journal focusing on cancer research and the optimal use of preventative and integrated treatment interventions to achieve improved outcomes, enhanced survival and quality of life for the cancer patient.

The manuscript management system is completely online and includes

Submit your manuscript here: https://www.dovepress.com/cancer-management-and-research-journal

a very quick and fair peer-review system, which is all easy to use. Visit http://www.dovepress.com/testimonials.php to read real quotes from published authors. 